\title{
ASCENT, DESCENT, AND COMMUTING PERTURBATIONS
}

BY

\author{
M. A. KAASHOEK AND D. C. LAY( $\left.{ }^{1}\right)$
}

\begin{abstract}
In the present paper we investigate the stability of the ascent and descent of a linear operator $T$ when $T$ is subjected to a perturbation by a linear operator $C$ which commutes with $T$. The domains and ranges of $T$ and $C$ lie in some linear space $X$. The results are used to characterize the Browder essential spectrum of $T$. We conclude with a number of remarks concerning the notion of commutativity used in the present paper.
\end{abstract}

Introduction. To discuss ascent and descent one must consider iterates of operators. Some sort of commutativity of $T$ and $C$ is necessary in order to meaningfully compare operators such as $T^{k}$ and $(T+C)^{k}$ and to "factor" operator products (cf. Lemma 1.4). We shall say a linear operator $C$ commutes with $T$ if (i) the domain of $C, \mathcal{D}(C)$, contains the domain of $T$, (ii) $C x \in \mathfrak{D}(T)$ whenever $x \in \mathscr{D}(T)$, and (iii) $T C x=C T x$ for $x \in \mathscr{D}\left(T^{2}\right)$. This definition coincides with the usual one when $T$ and $C$ are defined on all of $X$. Note that $T$ commutes with itself if and only if $T$ maps $\mathscr{D}(T)$ into $\mathscr{D}(T)$.

In $\$ 1$ we collect together a number of preliminary lemmas about operators $T$ and $C$ such that $C$ commutes with $T$. In $\$ 2$ we show by purely algebraic methods that the finiteness of the ascent or descent of $T$ is retained by the operator $T+C$ when $C$ commutes with $T$ and a certain power of $C$ has finitedimensional range. In general this does not hold if $C$ is a compact operator, but similar results may be obtained when some restrictions are placed on $T$. This is shown in $\$ 3$, where we consider perturbations by compact operators, Riesz operators and $T^{k}$-compact operators. In $\$ 4$ we use the results of $\$ 3$ to characterize the Browder essential spectrum. The main results of this section have been announced earlier by the second author (see [9]). In the final section we discuss the commutativity condition used here. Among other things we show that there exists a closed operator $T$ such that the only bounded operators commuting with $T$ are scalar multiples of the identity operator.

Received by the editors September 8, 1971 .

AMS 1970 subject classifications. Primary 47A10, 47A55, 47B05; Secondary 47B30.

Key words and phrases. Ascent, descent, finite-dimensional perturbations, compact perturbations, Riesz operators, Browder essential spectrum, commuting operatcrs.

(1) Research of the second author supported in part by the National Science Foundation Grant GP-12295. 
1. Algebraic properties of commuting operators. To deal with ascent and descent one has to consider iterates of an operator. Let $T$ and $C$ be linear operators with domains and ranges in the linear space $X$. The domains of $T$ and $C$ are denoted by $\mathscr{D}(T)$ and $\mathscr{D}(C)$ respectively. The product of $C$ and $T$ is the linear operator $C T$ with domain

$$
\mathfrak{T}(C T)=\{x \in \mathfrak{D}(T) \mid T x \in \mathfrak{D}(C)\}
$$

and defined by

$$
C T x=C(T x) \quad(x \in \mathfrak{D}(C T)) .
$$

Iterates of $T$ are now defined by induction. By definition, $T^{1}=T$, and for $n>1$ the operator $T^{n}$ is defined to be the product of $T$ and $T^{n-1}$, i.e. $T^{n}=T T^{n-1} . T^{0}$ shall be read as the identity operator on $X$.

In this section we collect together a number of lemmas about $T, T+C$ and their iterates.

1.1. Lemma. If $C$ commutes with $T$, then $-C$ commutes with $T+C$.

Proof. By definition, $\mathfrak{D}(T+C)=\mathfrak{D}(T)$, and thus $C \mathscr{D}(T+C) \subset \mathfrak{D}(T+C)$.

Take $x$ in $\mathscr{D}\left[(T+C)^{2}\right]$. Then $x \in \mathfrak{D}(T)=\mathfrak{D}(T+C)$ and $T x+C x=$ $(T+C)_{x} \in \mathfrak{D}(T+C)$. Also, $C x \in \mathscr{D}(T+C)$, so $T x \in \mathfrak{D}(T+C)=\mathfrak{T}(T)$, and $x \in \mathfrak{D}\left(T^{2}\right)$. From $C x \in C \mathscr{D}(T) \subset \mathfrak{D}(T) \subset \mathfrak{T}(C)$ we see that $x \in \mathfrak{D}\left(C^{2}\right)$. Thus we have

$$
(T+C) C x=T C x+C^{2} x=C T x+C^{2} x=C(T+C) x,
$$

that is, $-C$ commutes with $T+C$.

1.2. Lemma. Suppose that $C$ commutes with $T$. Then for $n=1,2, \cdots$,

(a) $C \mathfrak{I}\left(T^{n}\right) \subset \mathfrak{D}\left(T^{n}\right)$;

(b) $\mathcal{H}\left(T^{n}\right) \subset \Re\left(T^{n}\right)$, where $\Re\left(T^{n}\right)$ is the null space of $T^{n}$;

(c) $\mathfrak{D}(T) \subset \mathfrak{D}\left(C^{n}\right)$;

(d) $T^{n} C^{m} x=C^{m} T^{n} x$ for all $x$ in $\mathscr{D}\left(T^{n+1}\right)$ and $m=1,2, \cdots$;

(e) $(T C)^{n} x=T^{n} C^{n} x=C^{n} T^{n} x=(C T)^{n} x$ for $x$ in $\mathscr{D}\left(T^{n+1}\right)$;

(f) $\mathfrak{D}\left(T^{n}\right)=\mathscr{D}\left[(T+C)^{n}\right]$;

(g) $(T+C)^{n} x=\Sigma_{i=0}^{n}\left(\begin{array}{c}n \\ i\end{array}\right) T^{n-i} C^{i} x=\Sigma_{i=0}^{n}\left(\begin{array}{c}n \\ i\end{array}\right) C^{i} T^{n-i} x$ for $x$ in $\mathscr{D}\left(T^{n}\right)$.

Proof. The verification of the statements above by induction is straightforward (although somewhat tedious) and will be omitted.

Note that (a) and (d) of Lemma 1.2 together imply that $C^{m}$ commutes with $T$ for any $m$. This observation is useful in the proof of the next lemma.

Suppose that $T$ is one-one and onto. Then the inverse map $T^{-1}$ is well defined and $\mathcal{D}\left(T^{-1}\right)=X$. Observe that $T^{-1}$ commutes with $T$. For $n=1,2, \cdots$, we define $T^{-n}$ to be the $n$th iterate of $T^{-1}$, i.e. $T^{-n}=\left(T^{-1}\right)^{n}$. It is easily seen that $T^{-n}=\left(T^{n}\right)^{-1}$. 
1.3. Lemma. Suppose that $C$ commutes with $T$. If $T$ is one-to-one and maps $\mathcal{D}(T)$ onto $X$, then, for $n, m=1,2, \cdots$,

(i) $T^{-n} C^{m} x=C^{m} T^{-n} x$ for $x \in \mathscr{D}(T)$;

(ii) $\left(C T^{-1}\right)^{n} x=C^{n} T^{-n} x$ for $x \in X$.

Proof. Also omitted.

1.4. Lemma. Suppose that $C$ commutes with $T$. If $T$ is one-to-one and onto $X$, then, for $n=1,2, \cdots$,

$$
(T+C)^{n}=\left(I+C T^{-1}\right)^{n} T^{n}=T^{n}\left(I+C T^{-1}\right)^{n} .
$$

Proof. Since $\mathscr{D}\left(I+C T^{-1}\right)=X, \mathscr{D}\left[\left(I+C T^{-1}\right)^{n} T^{n}\right]=\mathscr{D}\left(T^{n}\right)$. By Lemma 1.3,

$$
\left(I+C T^{-1}\right)^{n} x=\sum_{i=0}^{n}\left(\begin{array}{l}
n \\
i
\end{array}\right)\left(C T^{-1}\right)^{i} x=\sum_{i=0}^{n}\left(\begin{array}{l}
n \\
i
\end{array}\right) C^{i} T^{-i} x .
$$

From Lemma 1.2(a) we conclude that $\left(I+C T^{-1}\right)^{n} x-x \in \mathscr{D}(T)$. Therefore, $x \in \mathscr{D}(T)$ whenever $\left(I+C T^{-1}\right)^{n} x \in \mathscr{D}(T)$. In this case, $C T^{-1} x \in \mathscr{D}\left(T^{2}\right)$, so that (2) implies $\left(I+C T^{-1}\right)^{n} x-x \in \mathscr{D}\left(T^{2}\right)$. Proceeding with the same argument, one sees that $x \in \mathscr{D}\left(T^{n}\right)$ whenever $\left(I+C T^{-1}\right)^{n} x \in \mathscr{D}\left(T^{n}\right)$. The converse is also true. Hence $\mathscr{D}\left[T^{n}\left(I+C T^{-1}\right)^{n}\right]=\mathscr{D}\left(T^{n}\right)$. In view of Lemma $1.2(f)$, this shows that the operators in (1) have the same domain.

Now given $x \in \mathscr{D}\left(T^{n}\right)$, we may write $x=T^{-n} w$ for some $w \in X$. From Lemma $1.2(\mathrm{~g})$ and (2), we have

$$
(T+C)^{n} x=\sum_{i=0}^{n}\left(\begin{array}{l}
n \\
i
\end{array}\right) C^{i} T^{n-i}\left(T^{-n} w\right)=\left(I+C T^{-1}\right)^{n} w=\left(I+C T^{-1}\right)^{n} T^{n} x .
$$

The verification of the second equality in (1) is similar.

2. Finite-dimensional perturbations. In this section $T$ and $C$ are linear operators with domains and ranges in the linear space $X$.

Following the notation and terminology of [16], we let $n(T)$ be the nullity of $T$, i.e. the dimension of the null space $\Re(T)$ of $T$; and we let $d(T)$ be the defect of $T$, i.e. the codimension of the range $R(T)$ of $T$. The ascent of $T, \alpha(T)$, is the smallest nonnegative integer $p$ such that $\Re\left(T^{p}\right)=\Re\left(T^{p+1}\right)$; if no such $p$ exists, we define $\alpha(T)=+\infty$. Similarly, the descent of $T, \delta(T)$, is the smallest nonnegative integer $q$ such that $R\left(T^{q}\right)=R\left(T^{q+1}\right)$, and $\delta(T)=+\infty$ if no such $q$ exists.

2.1. Lemma. Suppose that $C$ commutes with $T$. Then, for $k, n=1,2, \cdots$,

$$
\operatorname{dim} \frac{\Re\left(T^{n}\right)}{\Re\left[(T+C)^{n+k-1}\right] \cap \Re\left(T^{n}\right)} \leq \operatorname{dim} R\left(C^{k}\right) ;
$$




$$
\operatorname{dim} \frac{R\left(T^{n+k-1}\right)}{R\left[(T+C)^{n}\right] \cap R\left(T^{n+k-1}\right)} \leq \operatorname{dim} R\left(C^{k}\right) .
$$

Proof. Let $X_{1}$ be a subspace of $\eta\left(T^{n}\right)$ such that

$$
\Re\left(T^{n}\right)=\left\{\Re\left[(T+C)^{n+k-1}\right] \cap \Re\left(T^{n}\right)\right\} \oplus X_{1} .
$$

It follows from Lemma $1.2(g)$ that $(T+C)^{n+k-1}$ maps $\Re\left(T^{n}\right)$ into $R\left(C^{k}\right)$. Since $(T+C)^{n+k-1}$ is one-to-one on $X_{1}, \operatorname{dim} X_{1} \leq \operatorname{dim} R\left(C^{k}\right)$. This proves (a).

To prove (b), let $x_{1}, \cdots, x_{m}$ be in $\mathcal{T}\left(T^{n+k-1}\right)$ such that $T^{n+k-1} x_{1}, \cdots$, $T^{n+k-1} x_{m}$ are linearly independent in $R\left(T^{n+k-1}\right)$ modulo $R\left[(T+C)^{n}\right]$ $\cap R\left(T^{n+k-1}\right)$. Since $-C$ commutes with $T+C$ (Lemma 1.1), we may interchange $T$ and $T+C$ in Lemma 1.2(g). It follows that, for $i=1, \cdots, m$,

$$
T^{n+k-1} x_{i}=(T+C)^{n} u_{i}+C^{k} v_{i},
$$

for suitable $u_{i}$ and $v_{i}$ If $m>\operatorname{dim} R\left(C^{k}\right)$, then there exist constants $a_{1}, \cdots, a_{m}$ : not all zero, such that $\sum_{i=1}^{m} a_{i} C^{k} v_{i}=0$, and hence

$$
\sum_{i=1}^{m} a_{i} T^{n+k-1} x_{i}=\sum_{i=1}^{m} a_{i}(T+C)^{n} u_{i}
$$

Since the $a_{i}$ are not all zero, $\left\{T^{n+k-1} x_{i}\right\}_{i=1}^{m}$ is not linearly independent modulo $R\left[(T+C)^{n}\right] \cap R\left(T^{n+k-1}\right)$, a contradiction. Thus $m \leq \operatorname{dim} R\left(C^{k}\right)$, which proves (b).

2.2. Theorem. Suppose that $C$ commutes with $T$ and $\operatorname{dim} R\left(C^{k}\right)<\infty$ for some integer $k \geq 1$. Then, if $T$ bas finite ascent (resp. descent), $T+C$ bas finite ascent (resp. descent).

Proof. Suppose that $\alpha(T)=p<\infty$. For $n \geq p$, let

$$
\begin{aligned}
& a_{n}=\operatorname{dim} \frac{\pi\left(T^{n}\right)}{\pi\left[(T+C)^{n+k-1}\right] \cap \pi\left(T^{n}\right)}=\operatorname{dim} \frac{\pi\left(T^{p}\right)}{\pi\left[(T+C)^{n+k-1}\right] \cap \pi\left(T^{p}\right)}, \\
& b_{n}=\operatorname{dim} \frac{\pi\left[(T+C)^{n}\right]}{\pi\left[(T+C)^{n}\right] \cap \pi\left(T^{n+k-1}\right)}=\operatorname{dim} \frac{\pi\left[(T+C)^{n}\right]}{\pi\left[(T+C)^{n}\right] \cap \pi\left(T^{p}\right)} .
\end{aligned}
$$

By Lemma 2.1(a), $a_{n} \leq \operatorname{dim} \Re\left(C^{k}\right)<\infty$. Since the null spaces of the iterates of $T+C$ form an increasing nest of subspaces, it follows that there is an integer $N \geq p$ such that $a_{n}=a_{N}$ for $n \geq N$. But this implies

$$
\Re\left[(T+C)^{i}\right] \cap \Re\left(T^{p}\right)=\Re\left[(T+C)^{N+k-1}\right] \cap \Re\left(T^{p}\right)
$$

for $i \geq N+k-1$. By Lemma 1.1, we may interchange $T$ and $T+C$ in Lemma 2.1(a) to conclude that $b_{n} \leq \operatorname{dim} R\left(C^{k}\right)<\infty$. Clearly, $b_{n} \leq b_{n+1} \leq \cdots$ (for $n \geq p$ ), 
and there is an integer $M \geq N+k-1$ such that $b_{n}=b_{M}$ for $n \geq M$. When combined with (3) this implies that $\Re\left[(T+C)^{n}\right]=\Re\left[(T+C)^{M}\right]$ for $n \geq M$, i.e. $\alpha(T+C) \leq M<\infty$.

The proof for the case when $T$ has finite descent is similar and will be omitted.

Suppose that $C$ is a linear operator on $X$ with the following property: "If $T$ is a linear operator such that $C$ commutes with $T$ and $\alpha(T)<\infty$ and $\delta(T)<\infty$, then $a(T+C)<\infty$ and $\delta(T+C)<\infty$." Then, by taking $T$ to be scalar multiples of the identity operator, we see that

$$
\alpha(\lambda I+C)<\infty \text { and } \delta(\lambda I+C)<\infty
$$

for all $\lambda$. In the next section we will generalize Theorem 2.2 when $T$ is a closed linear operator on a Banach space $X$. If we try to consider a perturbation by a bounded operator $C$ (with $\mathscr{T}(C)=X$ ), then (4) implies that the spectrum of $C$ must consist of a finite set of poles of the resolvent operator (cf. Theorem 4.3 of [10]). In particular, (4) implies that Theorem 2.2 will not be true if " $C$ " has finite-dimensional range" is replaced by "C is compact". However, this result does hold if one places some restrictions on $T$. (This will be proved in the next section.)

It is an open question whether or not the class of operators having some iterate with finite-dimensional range is characterized by the property mentioned at the beginning of the preceding paragraph.

3. Compact perturbations. In this section $T$ and $C$ are linear operators with domains and ranges in the Banach space $X$, and $T$ is a closed operator. Note that we do not require $\mathscr{T}(T)$ to be dense in $X$.

\subsection{Proposition. Suppose that}

$$
n(T)=d(T)<+\infty \quad \text { and } \quad \alpha(T)<+\infty .
$$

Then there exists a bounded linear operator $B$ defined on $X$ with finite-dimensional range and such that

(a) $B T x=T B x$ for $x \in \mathscr{I}(T)$; in particular, $B$ commutes with $T$;

(b) $0 \in \rho(T+B)$, i.e. $T+B$ bas a bounded inverse defined on $X$;

(c) if $C$ commutes with $T$, then $C B x=B C x$ for $x \in \mathfrak{D}(T)$.

Proof. By Theorem 4.3 of [7],

$$
X=\Re\left(T^{p}\right) \oplus R\left(T^{p}\right)
$$

where $p=\alpha(T)$. Let $B$ be the projection of $X$ onto $\Re\left(T^{p}\right)$ along $R\left(T^{p}\right)$. Clearly, $B$ is defined on all of $X$ and $\operatorname{dim} R(B)<\infty$. Since $T$ is a closed linear operator with finite nullity and defect, $T$ is a Fredholm operator. Therefore $T^{p}$ is a Fredholm operator, and consequently $\Re\left(T^{p}\right)$ and $R\left(T^{p}\right)$ are closed subspaces of $X$. 
This means that $B$ is a bounded linear operator on $X$.

It is easy to verify that $B$ satisfies (a), (b), and (c).

It can be shown that the conditions in (5) imply that 0 is a pole of finite rank of the resolvent operator $(\lambda-T)^{-1}$. The proof of this for the case when $T$ is densely defined is contained in the proofs of Corollary 4 and Theorem 7 of [8] and in the proof of Theorem 2.1 of [10]. In those references the hypothesis $\overline{T(T)}=X$ was used to establish (6) and was not subsequently needed. The arguments in [8] and [10] therefore apply to the present situation, and will not be repeated here. The operator $B$ introduced in the proof of Proposition 3.1 is the spectral projection corresponding to the spectral set $\{0\}$ (cf. the proof of Lemma 2.7 in $[15])$.

3.2. Theorem. Suppose that $0 \in \rho(T)$, and let $C$ be a compact operator on $X$ which commutes with T. Then

$$
n(T+C)=d(T+C)<\infty \text { and } a(T+C)=\delta(T+C)<\infty .
$$

Proof. From Lemma 1.4 we see that, for $n=1,2, \cdots$,

$$
\Re\left[(T+C)^{n}\right]=\Re\left[\left(I+C T^{-1}\right)^{n}\right], \quad \mathscr{R}\left[(T+C)^{n}\right]=\mathscr{R}\left[\left(I+C T^{-1}\right)^{n}\right] .
$$

Now $C T^{-1}$ is compact since it is the product of a compact operator and a bounded operator. The theorem now follows from the Riesz theory for compact operators.

Theorem 3.2 would not be true if the condition that $C$ commutes with $T$ were removed. For example, let $X$ be the Banach space

$$
l_{1}=\left\{\left(\ldots, x_{-1}, x_{0}, x_{1}, \ldots\right)\left|\sum_{-\infty}^{\infty}\right| x_{i} \mid<\infty\right\} .
$$

Define $T$ on $X$ by

$$
(T x)_{n}=x_{n-1} \text { for } n=0, \pm 1, \pm 2, \cdots,
$$

and let $C$ be defined by

$$
(C x)_{n}= \begin{cases}0 & \text { for } n \neq 1, \\ x_{0} & \text { for } n=1 .\end{cases}
$$

Then $T$ is a bounded linear operator on $X, 0 \in \rho(T)$ and $C$ is a compact operator, but $\alpha(T+C)=\delta(T+C)=\infty$.

It is clear from the proof of Theorem 3.2 that the result of the theorem also holds if $C$ is a linear operator commuting with $T$ and such that $C T^{-1}$ has the same spectral properties as a compact operator, i.e. such that $C T^{-1}$ is a Riesz operator. Therefore the following lemmas are of interest. 
3.3. Lemma. Suppose that $0 \in \rho(T)$, and let $C$ be a Riesz operator on $X$ commuting with $T$. Then $C T^{-1}$ is a Riesz operator.

Proof. Consider the quotient algebra $[X] / \mathcal{K}$ of the bounded operators on $X$ modulo the compact operators, and use the well-known fact that an operator in $[X]$ is Riesz if and only if its spectral radius in $[X] / \mathcal{K}$ is zero. By Lemma 1.3, $\left(C T^{-1}\right)^{n}=C^{n} T^{-n}$, for $n=1,2,3, \cdots$. Viewing this equation in $[X] / \mathcal{K}$, it is easy to see that the image of $C T^{-1}$ in $[X] / \mathcal{K}$ has a spectral radius of zero.

A linear operator $C$ with $\mathscr{T}(C) \supset \mathscr{T}(T)$ is said to be $T^{k}$-compact (for $k$ some natural number) if, for any sequence $\left\{x_{n}\right\} \subset \mathscr{L}\left(T^{k}\right)$ satisfying $\left\|x_{n}\right\|+\left\|T^{k} x_{n}\right\| \leq$ const., the sequence $\left\{C x_{n}\right\}$ has a convergent subsequence. If $0 \in \rho(T)$ and $\mathscr{D}(C) \supset \mathscr{D}(T)$, it is not difficult to show that $C$ is $T^{k}$-compact if and only if $C T^{-k}$ is a compact operator on $X$ (cf. [3, p. 201]).

3.4. Lemma. Suppose that $0 \in \rho(T)$, and let $C$ be a $T^{k}$-compact operator commuting with $T$. Then $\left(C T^{-1}\right)^{k}$ is a compact operator on $X$; in particular $C T^{-1}$ is a Riesz operator.

Proof. We first prove that $C T^{-1}$ is a bounded linear operator on $X$. If $k=1$ then $C T^{-1}$ is compact and hence bounded. Suppose $k>1$. Let $\left\{y_{n}\right\}$ be a null sequence in $X$, and suppose that $C T^{-1} y_{n} \rightarrow w$ in $X$. Then by Lemma 1.3 and the boundedness of $T^{-(k-1)}$,

$$
C T^{-k} y_{n}=T^{-(k-1)}\left(C T^{-1} y_{n}\right) \rightarrow T^{-(k-1)} w .
$$

On the other hand, $C T^{-k}$ is bounded and so $C T^{-k} y_{n}$ must be a null sequence. This implies $w=0$. It follows that $C T^{-1}$ has a closed extension. Since the domain of $C T^{-1}$ is all of $X, C T^{-1}$ must itself be closed. But then $C T^{-1}$ is bounded by the closed graph theorem.

To show that $\left(C T^{-1}\right)^{k}$ is compact, we use Lemma 1.3 to see that

$$
R\left[\left(C T^{-1}\right)^{k}\right]=C^{k} \mathfrak{D}\left(T^{k}\right) \subset C \mathscr{D}\left(T^{k}\right)=C R\left(T^{-k}\right)=R\left(C T^{-k}\right) .
$$

Since $C$ is $T^{k}$-compact, $C T^{-k}$ is compact on $X$. Thus $\left(C T^{-1}\right)^{k}$ is a bounded operator whose range is contained in that of a compact operator. A result of Phillips implies that $\left(C T^{-1}\right)^{k}$ is compact (cf. Theorem 2.13 .8 of [6]).

3.5. Theorem. Suppose that $n(T)=d(T)<\infty$ and $\alpha(T)<\infty$. Let $C$ commute with $T$ and suppose that $C$ satisfies at least one of the following conditions:

(i) $C$ is a compact linear operator on $X$;

(ii) $C$ is a Riesz operator;

(iii) $C$ is $T^{k}$-compact.

Then $T+C$ is closed and 


$$
n(T+C)=d(T+C)<\infty \text { ind } a(T+C)=\delta(T+C)<\infty .
$$

Proof. By Proposition 3.1 there exists a bounded linear operator $B$ with finitedimensional range such that $0 \in \rho(T+B), B T x=T B x$ for $x \in \mathcal{T}(T)$, and $C B x=$ $B C x$ for $x \in \mathfrak{T}(T)$. It follows that $-B+C$ commutes with $T+B$. Furthermore, since $B$ is a compact operator,

(i)' if $C$ is compact, then $-B+C$ is compact;

(ii)' if $C$ is a Riesz operator, then so is $-B+C$;

(iii) ${ }^{\prime}$ if $C$ is $T^{k}$-compact, then $-B+C$ is $(T+B)^{k}$-compact.

To verify this last statement, we note that $(T+B)^{k}$ and $T^{k}$ are closed linear operators (see [4, Corollary IV.2.12]) with the same domain. Thus there exists a constant $M>0$ such that $\left\|T^{k} x\right\| \leq M\left(\|x\|+\left\|(T+B)^{k} x\right\|\right)$ for all $x$ in $\mathscr{D}\left(T^{k}\right)$. From this it follows that $C$ is $(T+B)^{k}$-compact. But $B$ is compact, so $-B+C$ is $(T+B)^{k}$-compact.

Next we observe that

$$
T+C=(T+B)+(-B+C) .
$$

Formula (7) now follows from Lemma 3.3, Lemma 3.4 and the remark preceding Lemma 3.3.

It remains to show that $T+C$ is closed. Without loss of ge nerality (see the first part of the proof) we may suppose that $0 \in \rho(T)$. We know that each of the conditions (i), (ii) and (iii) implies that $C T^{-1}$ is a bounded linear operator on $X$. By Lemma 1.4, $T+C=T\left(I+C T^{-1}\right)$. Since $T$ is closed, the last formula implies $T+C$ is closed.

The conclusions of Theorem 3.5 remain valid if the condition $\alpha(T)<+\infty$ is replaced by

$$
\mathscr{D}(T) \text { is dense in } X \text { and } \delta(T)<+\infty \text {. }
$$

To see this we note the following. From $n(T)=d(T)<+\infty$ and the fact that $\mathcal{T}(T)$ is dense in $X$, it follows that $T^{q}$ is a densely defined linear operator (see [4, Theorem IV.2.7(iv)]), where $q=\delta(T)$. Since $R(T)$ is closed and has finite codimension, it follows that $X=\mathscr{D}\left(T^{q}\right)+\mathscr{R}(T)$. But then $n(T)=d(T)<+\infty$ and $\delta(T)<+\infty$ imply that $\alpha(T)=\delta(T)<+\infty$ (see Theorem 4.6 of [7]). The observation made above now follows from Theorem 3.5.

Suppose that $C$ is a bounded linear operator with the following property: "If $T$ is a bounded linear operator such that $C$ commutes with $T, n(T)=d(T)<\infty$ and $\alpha(T)<+\infty$, then $n(T+C)=d(T+C)<\infty$ and $\alpha(T+C)=\delta(T+C)<\infty$." Then, by taking nonzero multiples of the identity operator, we see that

$$
n(\lambda I+C)=d(\lambda I+C)<\infty \text { and } a(\lambda I+C)=\delta(\lambda I+C)<\infty,
$$


for all $\lambda \neq 0$, i.e. $C$ is a Riesz operator. Thus condition (ii) in Theorem 3.5 cannot be weakened if $C$ is a bounded linear operator defined on all of $X$. This result is essentially Theorem 2.6 in [15].

It is not necessary to consider perturbations by $T$-pseudo-compact operators or by $T^{2}$-pseudo-compact operators (cf. [14]). This follows from the remarks following Theorem $S$ in $\$ 3$ of $[5]$.

4. Invariance of the essential spectrum. The results of the previous section can be used to give two characterizations of the essential spectrum of $T$, ess $(T)$, as defined by F. E. Browder [1]. It is well known (cf. [5], [14]) that if $T$ is a closed linear operator with domain and range in a Banach space $X$, then ess $(T)$ is the complement in the complex plane of the set

$$
\begin{array}{r}
\{\lambda \in \mathbf{C} \mid n(\lambda-T)=d(\lambda-T)<\infty \text { and a deleted neighbourbood of } \lambda \\
\text { is in the resolvent set of } T\} .
\end{array}
$$

Let $\mathcal{S}$ be a set of linear operators whose domains and ranges lie in $X$. We shall say that a subset $\Delta$ of the spectrum of $T, \sigma(T)$, remains invariant under perturbations of $T$ by operators in $\mathcal{S}$ if $\Delta \subset \bigcap_{S \in \mathcal{S}} \sigma(T+S)$.

In the present section we show that $\operatorname{ess}(T)$ is the largest subset of the spectrum which is invariant under compact and certain other commuting perturbations of $T$. The main results of this section have been announced earlier in [9].

4.1. Theorem. Let $T$ be a closed linear operator on a Banach space X. Then ess $(T)$ is the largest subset of the spectrum of $T$ wbich remains invariant under perturbations of $T$ by Riesz operators which commute with $T$.

Proof. Suppose that $\lambda \in \sigma(T) \backslash$ ess $(T)$. Then by Theorem 9.6 of [16], $\lambda$ is a pole of the resolvent operator, and Theorem 9.1 of [16] implies that $\alpha(\lambda-T)<\infty$. Substituting $T-\lambda$ for $T$ in Proposition 3.1, we see that there exists a bounded linear operator $B$ which commutes with $T-\lambda$ (and hence commutes with $T$ ), and $\lambda \notin \sigma(T+B)$. The operator $B$ is Riesz since it has finite-dimensional range.

On the other hand, suppose that $\lambda$ is in the spectrum of $T$ and there is a Riesz operator $B$ which commutes with $T$ such that $\lambda$ is not in the spectrum of $T+B$. Then $-B$ commutes with $T-\lambda+B$ by Lemma 1.1. Now

$$
n(T-\lambda+B)=d(T-\lambda+B)=0 \text { and } \quad a(T-\lambda+B)=0 .
$$

Then, by Theorem 3.5,

$$
n(T-\lambda)=d(T-\lambda)<\infty \text { and } a(T-\lambda)=\delta(T-\lambda)<\infty .
$$

Furthermore, $\mathscr{R}\left[(T-\lambda)^{p}\right\rceil$ is closed, since $T-\lambda$ is a Fredholm operator. Using Theorem 9.4 of [16] we can conclude that $\lambda$ is an isolated point of $\sigma(T)$. Thus $\lambda \notin \operatorname{ess}(T)$. 
It follows from the remarks at the end of the preceding section that the set of Riesz operators considered in Theorem 4.1 cannot be enlarged to contain any other bounded linear operator defined on all of $X$.

Theorem 4.1 remains true if "Riesz" is replaced by "compact" (this is the Corollary in [9]; see also Theorem $1(b)$ in [17], where this result is proved for bounded operators) or by " $T^{k}$-compact" (where $k$ depends on the perturbing operator). To see this we note that the operator $B$ used in the first paragraph of the proof of Theorem 4.1 is a bounded linear operator with $\mathcal{D}(B)=X$ and further $B$ has finite-dimensional range. This implies that $B$ is compact and $B$ is $T^{k}$ compact for any $k$. The rest of the argument is the same as the second part of the proof of Theorem 4.1, when "Riesz" is replaced by "compact" or " $T^{k}$-compact".

The fact that Theorem 4.1 remains true if "Riesz" is replaced by " $T$-compact" is somewhat surprising. Gustafson and Weidmann [5] have shown that, in general, for $k>2$ the Wolf essential spectrum of $T$ is not invariant under $T^{k}$ compact perturbations of $T$.

5. Remarks. In this section we present a number of remarks concerning the commutativity condition used in the present paper.

a. The requirement that $C$ commutes with $T$ is not in general a symmetric property. Caradus has given a definition of commutativity which does not have this deficiency (see Condition 3 in [2]). We have not used Caradus' definition, since it is not clear to us how one can compare $T^{k}$ and $(T+C)^{k}$ using his definition. Lemma 1.1 and Lemma $1.2(\mathrm{~g})$ are basic in the present paper. If they hold for an operator $C$ with $\mathscr{D}(C) \supset \mathscr{T}(T)$ and $C$ commuting with $T$ in the sense of Caradus, it would be desirable to use his definition.

b. Let $T$ and $C$ be linear operators with domains and ranges in the Banach space $X$, and let $T$ be a closed operator. It is interesting to note that under the conditions of Lemma 3.4 the $T^{k}$-compactness of $C$ implies that the map

$$
C: T(T) \rightarrow X
$$

is bounded in norm. The proof of this is based on the following lemma, which is due to R. D. Nussbaum.

5.1. Lemma. Suppose that $\rho(T) \neq \varnothing$ and let $C$ be a $T$-bounded operator commuting with $T$. Then the map (8) is bounded in norm.

Proof. See [13, Remark 3].

5.2. Proposition. Suppose that $\rho(T) \neq \varnothing$ and let $C$ be a $T^{\dot{k}}$-compact operator commuting with $T$. Then the map (8) is bounded in norm.

Proof. Without loss of generality we may suppose that $0 \in \rho(T)$. Then we 
know from Lemma 3.4 that $C T^{-1}: X \rightarrow X$ is bounded. Hence, for each $x$ in $\mathscr{I}(T)$,

$$
\begin{aligned}
\|C x\| & =\left\|C\left(T^{-1} T x\right)\right\|=\left\|\left(C T^{-1}\right) T x\right\| \\
& \leq\left\|\left(C T^{-1}\right)\right\| \cdot\|T x\| \leq\left\|\left(C T^{-1}\right)\right\|\{\|x\|+\|T x\|\} .
\end{aligned}
$$

This implies that $C$ is $T$-bounded. So we can use Lemma 5.1 to get the desired result.

c. The requirement that $C$ commutes with $T$ severely limits the class of perturbing operators. In the following we shall show that there exists a closed operator $T$ such that the only bounded operators commuting with $T$ are scalar multiples of the identity operator. The proof of this result is based on a variation of an argument used earlier by I. S. Murphy (see the proof of Theorem 1 in [11]).

We shall be dealing with weighted shifts on the Banach space $l_{1}$ of all absolutely convergent complex sequences (but the result we prove holds for any Banach space with a Schauder basis). An element of $l_{1}$ will be denoted by $x=$ $\left(x_{1}, x_{2}, \cdots\right)$.

Let $\left\{a_{n}\right\}$ be a sequence of nonzero complex numbers. We do not suppose that $\left\{a_{n}\right\}$ is a bounded sequence. Further, let $T$ denote the (possibly unbounded) weighted shift acting in $l_{1}$ with weights $a_{1}, \alpha_{2}, \ldots$. So the domain of $T$ is the set

$$
\mathfrak{T}(T)=\left\{x \in l_{1}\left|\sum_{n=1}^{\infty}\right| a_{n} x_{n} \mid<+\infty\right\},
$$

and on $\mathscr{D}(T)$ the operator $T$ is defined by the following formula:

$$
(T x)_{n}= \begin{cases}\alpha_{n-1} x_{n-1} & \text { for } n=1, \\ 0 & \text { for } n=1 .\end{cases}
$$

It is easy to see that $T$ is a closed linear operator with domain and range in $l_{1}$.

Let $e_{n}$ be the element in $l_{1}$ with all coordinates 0 except the $n$ th, which is equal to 1 . The following lemma is an immediate consequence of the definition of $T$; its proof will be omitted.

5.3. Lemma. For $n, k=1,2, \cdots$, the element $e_{n} \in \mathscr{T}\left(T^{k}\right)$ and $T^{k} e_{n}=$ $\alpha_{n} \alpha_{n+1} \cdots \alpha_{n+k-1} e_{n+k}$.

Now let $C$ be a bounded linear operator on $l_{1}$, commuting with $T$. Suppose

$$
C e_{1}=\left(\beta_{1}, \beta_{2}, \ldots\right) \text {. }
$$

5.4. Lemma. For $k=1,2, \cdots$, the element $C e_{1} \in \mathscr{D}\left(T^{k}\right)$ and

$$
T^{k} C e_{1}=\sum_{i=1}^{\infty} \alpha_{i} \alpha_{i+1} \cdots \alpha_{i+k-1} \beta_{i} e_{i+k} .
$$


Further, $T^{k} C e_{1}=C T^{k} e_{1}=\alpha_{1} \alpha_{2} \cdots \alpha_{k} C e_{k+1}$.

Proof. Since $e_{1} \in \mathfrak{D}\left(T^{k}\right)$ for any $k$, Lemma $1.2(a)$ shows that $C e_{1} \in \mathfrak{T}\left(T^{k}\right)$. Further, we can use Lemma $1.2(\mathrm{~d})$ to show that $T^{k} C e_{1}=C T^{k} e_{1}$. The remainder of the proof is a straightforward verification and will be omitted.

Note that Lemma 5.4 implies that the action of $C$ on $e_{k}(k=1,2, \ldots)$ is determined by $T$ and the action of $C$ on $e_{1}$. Since $C$ is bounded on $l_{1}$, it follows that $C$ is completely determined by $T$ and formula (9).

Put

$$
p_{m}(T)=\beta_{1} I+\sum_{j=1}^{m} \frac{\beta_{j+1}}{\alpha_{1} \alpha_{2} \cdots \alpha_{j}} T^{j} .
$$

Then $p_{m}(T)$ is a linear operator with domain $\mathscr{T}\left(T^{m}\right)$. So $p_{m}(T) e_{k}$ is well defined for $k=1,2, \cdots$. Note that $p_{m}(T) e_{1}=\Sigma_{i=1}^{m+1} \beta_{i} e_{i}$. For $k=1,2, \cdots$, we have

$$
\begin{aligned}
p_{m}(T) e_{k+1} & =\beta_{1} e_{k+1}+\sum_{j=1}^{m} \frac{\beta_{j+1}}{a_{1} \cdots \alpha_{j}} \alpha_{k+1} \cdots \alpha_{k+j} e_{k+1+j} \\
& =\frac{1}{\alpha_{1} \cdots a_{k}}\left(\sum_{j=0}^{m} \alpha_{j+1} \cdots \alpha_{k+j} \beta_{j+1} e_{k+1+j}\right) \\
& =\frac{1}{a_{1} \cdots \alpha_{k}}\left(\sum_{i=1}^{m+1} \alpha_{i} \cdots \alpha_{i+k-1} \beta_{i} e_{i+k}\right) .
\end{aligned}
$$

From these formulas we see that the sequence $\left\{p_{m}(T) e_{k} \mid m=1,2, \ldots\right\}$ is increasing in norm. Further, using Lemma 5.4, $\lim _{m \rightarrow+\infty} p_{m}(T) e_{k}=C e_{k}$ for $k=1,2, \cdots$. In particular, this implies that

$$
\left\|p_{m}(T) e_{k}\right\| \leq\|C\| \quad(k=1,2, \cdots) .
$$

The last formula shows that each $p_{m}(T)$ is bounded on the set $\left\{e_{1}, e_{2}, e_{3}, \cdots\right\}$. But then the same is true for

$$
\beta_{n+1} T^{n}=\alpha_{1} \alpha_{2} \cdots a_{n}\left\{p_{n}(T)-p_{n-1}(T)\right\},
$$

where $n=1,2, \cdots$.

Now suppose that $0<\left|a_{1}\right| \leq\left|a_{2}\right| \leq \cdots$ and that $\lim _{n \rightarrow+\infty}\left|a_{n}\right|=+\infty$. Then Lemma 5.3 shows that none of the powers of $T$ is bounded on $\left\{e_{1}, e_{2}, e_{3}, \cdots\right\}$. Combining this result with the conclusion of the previous paragraph, we see that $\beta_{n+1}=0$ for $n=1,2, \cdots$. Hence $C e_{1}=\beta_{1} e_{1}$. But then we can use Lemma 5.4 to show that $C e_{k}=\beta_{1} e_{k}(k=1,2, \cdots)$. This implies that $C$ is a multiple of the identity on $l_{1}$.

E. A. Nordgren [12] has proved that a Donoghue operator (i.e. a backwards shift with positive, montone, square summable weight sequence) on $l_{2}$ does not 
commute with any closed, unbounded linear operator. The present result seems to be a sort of inverse of Nordgren's result.

d. Suppose $T$ is a closed linear operator with a nonempty resolvent set. Then there always exist bounded operators commuting with $T$. Let $f \mapsto f(T)$ be the usual Dunford-Taylor operational calculus. It is easy to see that $f(T)$ is a bounded linear operator commuting with $T$ for any admissible function $f$.

\section{REFERENCES}

1. F. E. Growder, On the spectral theory of elliptic differential operators. I, Math. Aṇn. 142 (1961), 22-130. MR 35 \#804.

2. S. R. Caradus, Commutation properties of operator polynomials, J. Austral. Math. Soc. 12 (1971), 98-100.

3. I. C. Gohberg and M. G. KreY̌n, The basic propositions on defect numbers, root numbers, and indices of linear operators, Uspehi Mat. Nauk 12 (1957), no. 2 (74), 43-1 18; English transl., Amer. Math. Soc. Transl. (2) 13 (1960), 185-264. MR 22 \#3984.

4. S. Goldberg, Unbounded linear operators: Theory and applications, McGraw-Hill, New York, 1966. MR 34 \#580.

5. K. Gustafson and J. Weidmann, On the essential spectrum, J. Math. Anal. Appl. 25 (1969), 121-127. MR 39 \#3339.

6. E. Hille and R. S. Phillips, Functional analysis and semi-groups, rev. ed., Amer. Math. Soc. Colloq. Publ., vol. 31, Amer. Math. Soc., Providence, R.I., 1957. MR 19, 664.

7. M. A. Kaashoek, Ascent, descent, nullity and defect: A note on a paper by A. E. Taylor, Math. Ann. 172 (1967), 105-115. MR 36 \#5719.

8. - On the Riesz set of a linear operator, Nederl. Akad. Wetensch. Proc. Ser. A 71 = Indag. Math. 30 (1968), 46-53. MR 37 \#2025.

9. David Lay, Characterizations of the essential spectrum of F. E. Browder, Bull. Amer. Math. Soc. 74 (1968), 246-248. MR 36 \#4370.

10. - Spectral analysis using ascent, descent, nullity and defect, Math. Ann. $184(1969 / 70), 197-214$. MR 41 \#4279.

11. I. S. Murphy, Non-compact operators that act compactly on their centralisers, Bull. London Math. Soc. 2(1970), 307-312.

12. Eric A. Nordgren, Closed operators commuting with a weighted shift, Proc. Amer. Math. Soc. 24 (1970), 424-428. MR $41 \# 2435$.

13. Roger D. Nussbaum, Spectral mapping theorems and perturbation theorems for Browder's essential spectrum, Trans. Amer. Math. Soc. 150 (1970), 445-455.

14. M. Schechter, On the essential spectrum of an arbitrary operator. I, J. Math. Anal. Appl. 13 (1966), 205-215. MR 32 \#6230.

15. - On perturbations of essential spectra, J. London Math. Soc. (2) 1 (1969), 343-347. MR $40 \# 1809$.

16. A. E. Taylor, Theorems on ascent, descent, nullity and defect of linear operators, Math. Ann. 163 (1966), 18-49. MR 32 \#8169.

17. Kenneth K. Warner, The spectrum of a linear operator under perturbation by certain compact operators, Proc. Amer. Math. Soc. 22 (1969), 667-671. MR 39 \#7462.

WISKUNDIG SEMINARIUM, VRIJE UNIVERSITEIT, AMSTERDAM, THE NETHERLANDS

DEPARTMENT OF MATHEMATICS, UNIVERSITY OF MARYLAND, COLLEGE PARK, MARYLAND 20740 